\title{
La catástrofe demográfica indígena ${ }^{\mathrm{A}}$
}

\section{Germán Colmenares}

\author{
Quisiera comenzar esta charla ${ }^{\mathbf{B}}$ recordando el clima en el cual se dieron los debates \\ sobre los efectos provocados por el impacto de la Conquista, en las grandes culturas \\ americanas.
}

Conferencia dictada en el Museo del Oro del Banco de la República en Bogotá en el primer semestre de 1989. Transcripción y notas de Augusto Gómez, historiador, profesor de la Universidad Nacional de Colombia. Augusto Gómez es además el autor de todas las notas que aparecen aquí precedidas de la frase: "Nota del Editor". Colmenares conservaba dentro de sus "papeles" el texto que le había enviado con la transcripción de la exposición el profesor Gómez, académico al que el historiador apreciaba no solo como colaborador en algunas de sus investigaciones, sino como amigo. Según el recuerdo de Augusto Gómez, quien grabó la conferencia, su organización fue más bien incidental — se aprovechó la presencia de Colmenares en la ciudad, mientras preparaba su ingreso a la Universidad Nacional de Colombia-, y el público asistente estuvo compuesto por estudiantes de las universidades Nacional y de los Andes, y un grupo reducido de profesores universitarios y de funcionarios del Museo del Oro. Colmenares, quien no había preparado nada para la ocasión, se dejó llevar por sus conocimientos adquiridos y habló en el nivel de divulgación para un público no especializado, aunque sí interesado el propio expositor se refiere a su intervención como "charla" y no hace uso de la palabra conferencia-. Todas las notas presentadas en números arábigos fueron puestas en su momento por el profesor Augusto Gómez. Hay que recordar que tales notas tenían un carácter provisional, y en esta transcripción no han sido retocadas ni actualizadas. No solo no reflejan el estado actual de nuestros conocimientos sobre el tema, sino que tampoco agotan los conocimientos normales en esos años sobre el problema. El texto transcrito fue enviado a Colmenares primero por aprecio, y segundo, seguramente, para que diera una primera opinión sobre él, pero no sabemos si el autor pudo revisar la transcripción. Lo más seguro es que el autor de la "charla" no la hubiera publicado bajo esa forma. Lo incluimos, entre varias razones, para recordar la permanencia del "problema indígena" en las preocupaciones de Colmenares, y en general de la mayor parte de los miembros de la llamada Nueva Historia, comenzando por su propio inspirador, el profesor Jaime Jaramillo Uribe. Una preocupación más que justa, que en los años finales de su vida de Colmenares se había vuelto a convertir de manera visible en uno de sus grandes focos de interés, como lo recuerda su texto de ingreso como miembro correspondiente de la Academia Colombiana de Economía: "La aparición de la economía política de las Indias", texto del que existen publicadas varias versiones de revista y libro, pero que por alguna razón que no conocemos, no fue incluido en la edición de llamada Obra completa del autor.

B El lector debe mantener en su "espíritu", mientras lee, cuáles son irremediablemente las limitaciones de toda exposición oral, el mal papel que cumplen las muletillas, las repeticiones, las frases de cajón y el desgreño que se introduce en nuestras exposiciones orales, todo ello aquí presente, aunque posiblemente en menor grado que el que es corriente en las clases universitarias que, como se sabe, son en su mayoría ejemplo insuperable de improvisación. Hay grabadas en varios archivos y bibliotecas del país, por ejemplo en la Biblioteca Luis Ángel Arango, exposiciones más o menos formales de Germán Colmenares, pero ninguna de las que conocemos parece escapar a las dificultades que plantea toda exposición oral, y hay también exposiciones que son una combinación de un texto escrito con palabras improvisadas durante la exposición, y cuyo resultado final no fue revisado por el autor. Hay que recordar que en la cultura colombiana de la segunda mitad del siglo XX la publicación de "charlas" o conferencias grabadas a autores de cierta importancia, y publicadas sin revisión del autor - casi siempre post mortem —, constituye una experiencia no siempre ejemplar, y en ocasiones ha contribuido a "popularizar" textos que no es seguro que sus propios autores hubieran aprobado. 
Este debate se originó, evidentemente, en un ambiente de pugnacidad ideológica en los siglos XVI y XVII. El hecho de que España en cierta manera defendiera una política anacrónica; el hecho de que Carlos V llevara sus ejércitos a todos los rincones de Europa pasa sostener ese ideal medieval de la unidad cristiana, de la ortodoxia romana, generalmente se prestaba, particularmente dentro de estas controversias del siglo XVII, a ataques furibundos con respecto a la obra de España en América. Fue así como dentro de este clima, los escritos del Padre Las Casas sirvieron de cierta manera, para aportar los argumentos que "demostraban" a los detractores de España, que esta

potencia, que defendía una ortodoxia católica, había causado un impacto y unas consecuencias catastróficas en las poblaciones del Nuevo Mundo ${ }^{1}$. Como sabemos, este fue el origen de la llamada "leyenda negra"2.

Sin embargo, no siempre la obra lascasiana produjo las reacciones que habían puesto en tensión a "las dos Españas". A finales del siglo XIX en algunos países hispanoamericanos, particularmente en Colombia, por razones de la constitución de las "nuevas nacionalidades", se produjo un sentimiento de hispanofilia que se opuso a la "leyenda negra" que había caracterizado en el pasado la presencia de España en el Continente.

Es así como podríamos pensar que en Colombia la nacionalidad desde el punto de vista ideológico se fundamente en cuatro pilares que en cierta manera son: la gramática, la hispanidad — con lo que la hispanidad representa-, el lenguaje y la religión. Esta conservadurización del país lleva a ciertas posiciones extremas de hispanofilia (recordemos, pues, a todos nuestros gramáticos presidentes, el famoso regalo del tesoro Quimbaya a la reina Cristina), bastante subida, que crea una antítesis a la vieja "leyenda negra", para dar lugar a la "leyenda rosa", montada sobre todo sobre interpretaciones de tipo institucional, de tipo jurídico, que ven en las Leyes de Indias y en la misma Recopilación..., por ejemplo, un monumento a la sabiduría y al humanismo español.

$1 \quad$ Nota del editor: La obra de Fray Bartolomé de las Casas, a la cual se refería el historiador Germán Colmenares en esta conferencia, está compuesta por los "Tratados", y entre estos, la "Brevíssima relación de la destruyción de las Indias", publicada originalmente en 1522, en la imprenta de Sebastián Trujillo. Sin embargo y pese al reconocimiento que se ha hecho a la "Brevíssima relación" por parte de la mayoría de los historiadores de la Península y de los americanistas, tenemos que aceptar que toda la obra lascasiana se desenvuelve alrededor de los efectos negativos que causó la presencia de España en América y, que en estas circunstancias, no podemos circunscribirnos únicamente a la obra ya nombrada. Consultar: "De único vocationis modo ómnium Gentium ad veram religionem"; la "Historia General de las Indias" y la "Apologética Historia".

2 Nota del editor: Las ediciones que se hicieron de la "Brevíssima relación" fuera de España, ponen de manifiesto lo álgido del debate que se suscitó en esos años y las tensiones existentes entre las diversas monarquías europeas, en ese entonces. Se hicieron 15 ediciones holandesas, 13 francesas, 7 inglesas, 6 alemanas, 3 italianas, 3 latinas, 1 portuguesa y 1 japonesa. En cambio, las ediciones españolas presentan una tendencia claramente diferente con las anteriores. En efecto la primera edición se hizo en 1552, la segunda en 1646 y las siguientes serán de nuestra época. 
Creo que estas controversias en nuestros tiempos, particularmente después de los trabajos que se hacen en Berkeley, que comienzan pues con el libro famoso de Carl Ortwin Sauer ${ }^{3}$ y más tarde de Lesley Byrd Simpson ${ }^{4}$ y particularmente Woodrow Borah $^{5}$, quien explora sistemáticamente lo que se llama en México "La suma de visitas", permiten (antes que una reconstrucción impresionista del tipo, por ejemplo, de la de Ángel Rosenblat ${ }^{6}$, quien había utilizado a los cronistas como fuente), mediante el método estadístico de la extrapolación, hacernos a una imagen o a una idea más precisa del estado de la población aborigen en los años finales del siglo XV y durante los siglos XVI a XVIII. Estos estudios pioneros que se hicieron en Berkeley, y que se han repetido a través de trabajos similares en el Perú, en la provincia de Quito y en casi todas las regiones de Suramérica, permiten una visión un poco diferente sobre el problema demográfico de la América española.

Quiero recordar que las cifras de Borah cuando fueron publicadas crearon un escándalo y conmovieron hasta sus cimientos el mundo académico y científico de mediados de los años cincuenta y sesenta del presente siglo XX. Realmente, afirmar que

3 Nota del Editor: Germán Colmenares hace referencia al libro de Sauer, The Early Spain Main (Berkeley y Los Ángeles: 1966).

4 Nota del Editor: G. Colmenares se refiere a "The population of towns in Michoacán in 1554", Hispanic American Historical Review, 30 (1950): 248-250.

$5 \quad$ Nota del editor: La obra de Woodrow Borah sobre las poblaciones nativas que habitaban en el continente americano a la llegada de los españoles es extensa, la mayor parte escrita en lengua inglesa. Podemos destacar: "The Cathedral Archive of Oaxaca", Hispanic American Historical Review (1948): 640-645; "The collection of tithes in the bishopric of Oaxaca during the sixteenth century", Hispanic American Historical Review, 21 (1941): 386-409; "La defensa fronteriza durante la gran rebellion tepehuana", Historia Mexicana 16, n. ${ }^{\circ} 61$ julio-septiembre (1966): 14-29; "Francisco de Urdiñola's census of the Spanish settlements in Nueva Vizcaya, 1604", Hispanic American Historical Review 35 (1955): 398-402; Paul Deprez, comp., The Historical demography of Latin America: sources, techniques, controversies, fields, in population and economics. Proceedings of Section V of The International Economic History Association, 1968 (Winnipeg: University of Manitoba Press, 1970), 173-205; "Tithe collection in the Bishopric of Oaxaca, 1601-1867", Hispanic American Historical Review 29 (1949): 498-517; Bernardo García Martínez et al., comps., "Los tributos y su recaudación en la Audiencia de Nueva Galicia durante el siglo XVI", Historia y Sociedad en el Mundo de Habla Española. Homenaje a José Miranda (México: 1970), 27-47. W. Borah publicó también junto con Sherburne F. Cook, "The Aboriginal Population of central Mexico on the eve of the Spanish Conquest", Ibero-Americana 45 (1963); "Marriage and legitimacy in Mexican culture: Mexico y California", California Law Review 54, n. 2 (1966): 965-979, 996-1002; "The population of Central Mexico in 1548: an analysys of tuhe 'Suma de visitas de pueblos", Ibero-Americana 40 (1960); "Price trends of some basic commodities in Central Mexico, 1531-1570", Ibero-Americana 40 (1960). De estos investigadores fueron publicados en español: Ensayos sobre historia de la población. México y el Caribe, tomos 1 y 2 (México: 1977 y 1978). Ensayos sobre historia de la población: México y el Caribe. t. 3 (México: 1980). En el tomo 1 de la colección publicada en español se encuentra un capítulo dedicado a analizar los aportes de Juan Friede y Germán Colmenares a la historia demográfica, titulado: "La demografía histórica de las tribus de Colombia, en los estudios de Juan Friede y Germán Colmenares".

6 Nota del Editor. De este autor fueron publicadas: La población de América en 1492. Viejos y nuevos cálculos (México: 1967), y La población indígena y el mestizaje en América (Buenos Aires: 1954), 2 vols. 
en el área central de México había 20 millones de habitantes, tenía unas implicaciones tremendas. Pero quisiera también subrayar el hecho de que lo que se trata en este caso no es de hacer juicios morales sobre los conquistadores o sobre su presunta crueldad o sed de oro, o hacer una imagen como la hubiera hecho Prescott ${ }^{7}$ en el siglo XIX, sino que se trata de mirar objetivamente el problema demográfico.

Yo he tratado a través de estos años de estudiar un poco este problema con las cifras que proporcionan las "Visitas" y comprender objetivamente y por fuera de todo moralismo los procesos que condujeron a la llamada "catástrofe demográfica". Sin embargo, aunque la intención y el objetivo son muy claros, el camino a seguir no es fácil y muchas veces no nos recompensa el tiempo invertido en el examen de estas cifras. Sabemos por ejemplo, que aunque los fondos de "Visitas" del Archivo Nacional [actual AGN de Colombia] todavía son muy ricos y están en parte inexplorados, este hecho nos crea una dificultad, pues carecemos de una mirada global que de alguna manera nos señale las tendencias generales de las poblaciones nativas en los siglos XVI y XVII, y los efectos y desajustes que se produjeron en ellas como consecuencia del contacto con los miembros de las huestes españolas que invadían y penetraban sus territorios en ese entonces.

Esta carencia de una visión globalizada del problema demográfico en los años iniciales de la empresa de conquista y colonización ha actuado para que no podamos descifrar los mecanismos más sutiles que se emplearon para sujetar a unos hombres al yugo de otros, para provocarles un "desgano total" y para afectar sus tendencias de crecimiento de población en el corto plazo y en la larga duración.

Recuerden ustedes que el primero que exploró este campo en Colombia fue Orlando Fals Borda en su libro El hombre y la tierra en Boyacá, y luego en Campesinos de los Andes ${ }^{8}$; posteriormente Juan Friede y Jaime Jaramillo Uribe ${ }^{9}$ abordaron más

$7 \quad$ Nota del editor: La referencia de Colmenares es a William Prescott, cuya obra principal se sintetiza en dos grandes publicaciones: Historia de la Conquista del Perú - reimpresa en español en la Argentina en 1943- e Historia de la Conquista de México, que fue publicada en español, en la Argentina, en 1944.

8 Nota del editor: El hombre y la tierra en Boyacá de Orlando Fals Borda fue publicada inicialmente en 1957, en Bogotá, en las "Ediciones de documentos colombianos". La segunda edición de este libro, revisada y actualizada, fue publicada en 1973, en Bogotá, en las ediciones Punta de Lanza. Campesinos de los Andes del mismo Orlando Fals Borda, apareció en castellano en 1961, pero su edición original en inglés corresponde al año 1955. Campesinos de los Andes es la tesis de maestría del profesor Fals Borda y El Hombre y la tierra en Boyacá es su tesis doctoral.

9 Nota del editor: El artículo de Juan Friede a que se refiere el profesor Germán Colmenares fue publicado a principios de los años sesenta: "Algunas consideraciones sobre la evolución demográfica en la provincia de Tunja", Anuario Colombiano de Historia social y de la Cultura, n. ${ }^{\circ}$ (1965). Los trabajos citados de Jaime Jaramillo Uribe son "La población indígena de Colombia en el momento de la conquista y sus posteriores transformaciones", Anuario Colombiano de Historia Social y de la Cultura, n. 2 (1964) 239-295: y "Cambios demográficos y aspectos de la política social española en el Nuevo Reino de Granada durante la segunda mitad del siglo XVIII", en La personalidad histórica de Colombia y otros ensayos (Bogotá: Instituto Colombiano de Cultura, 1977), 167-179. 
sistemáticamente los efectos de la "catástrofe demográfica" en el periodo colonial y en relación con los grupos étnicos y el proceso de mestización; finalmente, algunos trabajos que se hicieron en la "Escuela de Sevilla"10, utilizando también resúmenes de vistas que se encuentran en el archivo de esta ciudad [AGI, Sevilla]. Así y todo, el fondo de "Visitas" no se ha explotado sistemáticamente y no se han aprovechado las inmensas posibilidades que brinda para conocer el estado de las poblaciones indígenas en este periodo.

Las visitas que se hicieron (recuerden ustedes la institución de la visita de la tierra que se estableció a mediados del siglo XVI en momentos de fundar la Audiencia [en Santafé], la cual implicaba para los oidores la obligación de salir periódicamente a "visitar la tierra"), sabemos que tenían por objeto realmente tasar los tributos. La tasa de tributos significaba en esencia una lucha política entre la Corona española, que se había desprendido (mediante lo que podríamos llamar un "sistema de contratación privada" con los "Adelantados", con los hombres que hacen la conquista) de una serie de atributos que hoy serían atribuidos a la soberanía del Estado. Uno de esos atributos naturalmente es el que conlleva el vasallaje: los vasallos. Se buscaba que el excedente económico que producían los vasallos pasara, a través de canales de reactivas [ [i?] de poder, hasta la cúpula, en este caso el rey de España.

Ahora bien, el siglo XVI fue un periodo de luchas políticas en torno a estas pretensiones de los "beneméritos", los conquistadores, que no quisieron dejarse quitar este atributo o este privilegio, pactado originalmente en casi todas las actas, en todas las llamadas "Capitulaciones", con los conquistadores; privilegio que significaba la posibilidad de hacer que el excedente económico que producían sociedades estables, es decir las grandes civilizaciones americanas, pasara, a través de canales diferentes al poder, al Estado. Estos canales estuvieron conformados por personas que actuaron como intermediarios, quienes no quisieron en la práctica atender los requerimientos tributarios de la Corona. Sabemos por ejemplo que ellos se negaron siempre a pagar el "quinto real"; sabemos que tuvieron una serie de conflictos con las Leyes Nuevas de 1542, que limitaban las encomiendas por dos vidas. Muchas de las consecuencias de esta actitud se manifestaron en las guerras del Perú y en parte en la Nueva Granada. En esta última, como sabemos, hubo también cierto remezón y tuvieron que ser suspendidas.

Aunque estas leyes se eludieron sistemáticamente por parte de los protagonistas de los hechos de la conquista y colonización, en general el esfuerzo de la Corona

10 Nota del Editor: Los trabajos de la llamada "Escuela de Sevilla" a los que se refiere aquí el profesor Colmenares fueron publicados por la Escuela de Estudios Hispanoamericanos de Sevilla. Ellos son: Silvia Padilla Altamirano, "Tasaciones de encomiendas en Popayán en el siglo XVI"; María Luisa López Arellano, "Las encomiendas de Popayán en los siglos XVII y XVIII"; Adolfo Luis González Rodríguez, "La sociedad encomendera en la Gobernación de Popayán durante el siglo XVII", en La encomienda en Popayán (Tres estudios) (Sevilla: Escuela de Estudios Hispanoamericanos de Sevilla, 1977). 
siempre estuvo dirigido a tasar el tributo, es decir a establecer una cantidad fija, en cierta medida razonable, para evitar que el encomendero el pago del tributo en un sistema de trabajos forzados.

Es por ello por lo que, a través de estas visitas, que son en cierta manera el resultado de luchas políticas, hoy en día nos atrevemos a dar una idea general de la evolución de la población indígena y de la tendencia de los movimientos en el largo plazo.

Ahora bien, creo que hay unas cuantas demostraciones que son muy simples desde el punto de vista estadístico, y que nos sirven para extrapolar hacia los años iniciales de la conquista el primer impacto, que se supone produjo uno mucho más grande en los años posteriores, expresado en una curva cuyo perfil tiende a mostrarnos incrementos más marcados en la mortalidad indígena, en la medida en que se fue consolidando el sistema colonial.

Digamos que en las visitas de la Costa [Caribe], en las visitas de Santander, en las visitas de la provincia de Popayán, y sobre todo en las visitas que se hicieron en el centro [actual] del país, a partir de la época de Venero de Leyva, pasando por las grandes visitas de Lesmes, Espinosa, Sarabia y de Luis Enríquez a finales del siglo XVI, comparando con los datos de mediados del siglo XVI a mediados del siglo XVII, nos da siempre una curva que tiene un perfil específico. Una curva en la cual, tomada la frecuencia de los puntos de visita, nos permite ver que el patrón del impacto de la dominación española es muy similar en toda América, y que presenta solo unas [pocas] variantes. Por ejemplo, se calcula que las tasas de crecimiento de las poblaciones indígenas tuvieron variaciones enormes dependiendo de si estaban localizadas en tierras calientes o en tierras frías.

En general estos datos son bastantes conocidos y el problema radica, a mi manera de ver, en la interpretación que pueda darse de este fenómeno, es decir, a la explicación que pueda darse de este hecho empírico que creo que está suficientemente documentado.

A pesar de que podemos discutir un poco sobre la variedad y las cifras de nuestra población, la materia de base se encuentra en las visitas.

Particularmente en los trabajos de Borah, que toma la Suma Visita efectuada en 1540, se aprecia el alcance de los efectos demográficos de la presencia de España en el suelo americano. El investigador tuvo que extrapolar cerca de 20 años y fue esa extrapolación la que le permitió llegar a la cifra de 20 millones de habitantes iniciales en el Valle Central de México, lo cual a algunos les ha parecido exagerado. Piensan entonces que ahí debe haber algo raro.

Pero aun sin llegar a una hipótesis tan atrevida, el descenso ya es tan regular, tan sistemático, que nos impele de alguna manera a dar una interpretación, y de hecho estas son muy variadas.

El mismo Borah se inclina, por ejemplo, a pensar en el impacto de las enfermedades pulmonares. Recuerden ustedes que la Corona siempre prohibió sacar a los 
indígenas de su [medio] natural, porque le parecía que sacar indígenas de una altura de 2000 metros y levarlos al nivel del mar, o viceversa, incrementaba su mortalidad. Por ejemplo, en el caso de Pamplona, cuando descubrieron las minas de esta localidad, después de 1540, se llevaron indígenas de las comunidades chibchas de la región de Tunja, de la región de Santafé; se llevaron a Pamplona, y naturalmente allí hubo una mortandad enorme. Lo mismo sucedió al llevarlos, por ejemplo, del Río de Oro hacia Vetas, una región alta. Esto producía, pues, un colapso pulmonar en muchos casos.

Pero hay también quienes se inclinan a pensar que fue la viruela; otros creen que posiblemente fue un fenómeno producido por las pocas oportunidades de contacto sexual, por los trabajos a que fueron sometidos los indígenas desde su más tierna edad.

Por el contrario, yo he preferido realmente visualizar el problema dentro de un esquema global, dentro de una totalidad. Quiero aclarar mi idea. Si pensamos en la estructura [social] indígena, y decimos o suponemos que hay una base biológica en ella que se encuentra en el fundamento de toda pirámide social, de toda estructura social, que hay unas organizaciones familiares y sociales, que hay unas estructuras políticas, y que todo esto tiene ápice en lo que podríamos llamar las mentalidades, entonces podríamos concluir, al menos provisionalmente, que los efectos de la hispanización de este Continente impactaron a las sociedades indígenas en su conjunto y no en el nivel de sus miembros tomados individualmente.

Antes de seguir adelante considero que debo dar una explicación. Por mentalidades no me refiero solamente a lo que podríamos llamar superestructura en términos marxistas; mucho más amplia [es la idea]. No sería religión... serían esas apoyaturas..., todos los códigos, esos códigos culturales de los que habla Geertz ${ }^{11}$, que son los que permiten la interpretación de la sociedad. Es decir, este ápice, y suponemos que esta es la totalidad de la estructura social. Lo que podemos postular es que realmente la Conquista produjo un impacto sobre todos y cada uno de los niveles [de la sociedad] y lo hizo simultáneamente.

Entonces, yo creo que el problema de tratar de explicarnos cómo, dentro de la tecnología de la muerte del siglo XVI, se produjo un impacto tan espeluznante, deja de aparecer tan misterioso, porque realmente la cifra que trae Borah de los $20 \mathrm{mi}-$ llones, es un guarismo que nos hace pensar, y sobre todo comparar situaciones que, guardadas las distancias, se asemejarían por lo menos en sus efectos devastadores con aquellos que se dieron hoy hace quinientos años.

Los alemanes en la Segunda Guerra Mundial, con medios técnicos muy sofisticados, exterminaron seis millones de judíos. ¿Cómo es que los españoles con medios técnicos tan elementales pudieron exterminar a más de 20 millones de nativos?

11 Nota del editor: La referencia de G. Colmenares es a Clifford Geertz, The Interpretation of Cultures (New York: Basic Books, 1973). 
Nos queda claro solamente algo que las generaciones de colombianos hemos podido captar. Tenemos más o menos idea de lo que significan estos descalabros sociales en lo que un antropólogo ha dado en llamar recientemente los "espacios de la muerte" 12 . Aunque estamos bastante familiarizados con esto, aun parecen ser para nosotros unas cifras excesivas.

En el caso del Nuevo Reino de Granada, podemos pensar que la población indígena oscilaba entre 3 y 6 millones, de acuerdo con el tipo de extrapolación que se haga, y que finalmente, hacia finales del siglo XVI, habría apenas unos 200 mil indígenas chibchas. Entonces fue un decrecimiento terminal correspondiente al $90 \%$ de la población indígena original. ¿Cómo ocurrió esto? A mi modo de ver la interpretación no debe poner énfasis en los aspectos de tipo biológico, en aspectos de tipo familiar o estructura social, sino que tendría que examinarse como una totalidad simultánea que ataca todos estos niveles y que naturalmente produce como resultado final un impacto demográfico.

Quisiera referirme simplemente uno por uno a estos niveles. Pensemos un poco en lo que ocurre con los aspectos biológicos. Creo que hay una serie de curvas muy interesantes que muestran una correlación entre el crecimiento del ganado vacuno y el decrecimiento de la población indígena. Una correlación muy estrecha entre estos dos fenómenos nos lleva a interrogarnos en términos concretos sobre lo que significó la conquista con respecto a la adaptación del espacio. Me atrevería a afirmar que en un país como Colombia, y creo que esto es igualmente cierto para México y el Perú, la famosa colonización española no fue colonización en el sentido de que los españoles ocuparon siempre las tierras roturadas por los indígenas.

Si ustedes piensan, por ejemplo, en los datos que nos legó Agustín Codazzi, él dice que la 1/5 partes del país estaban deshabitadas para la época en que lo recorrió. Esto es llamativo si pensamos que el proceso de colonización, digamos de desplazamiento de población mestiza hacia las vertientes, es un fenómeno del siglo XIX.

Piensen ustedes, por ejemplo, que los claustros coloniales se desarrollan en ciudades como Pasto, Almaguer, Cali, Caloto, Buga, Toro, Cartago, Anserma, por el occidente, y luego por el oriente; y así tienen ustedes un árbol que se ramifica: Santafé, Tunja, Vélez, Pamplona, Ocaña, Mérida. De resto los territorios fueron de indios. Pero así mismo vemos que prácticamente estos poblamientos originales de los españoles abarcaron regiones extensas: la jurisdicción real de una ciudad como Pasto, por ejemplo, comprendía el antiguo espacio de los Quillacingas; en el caso de Santafé y Tunja, el antiguo espacio Chibcha; en Vélez y Pamplona, los Guane, principalmente. Entonces fueron espacios que no se acabaron de llenar.

12 Nota del editor: no existe aún traducción al español de la obra de Michael Taussig, a la que Colmenares hace referencia. Para comprender su afirmación sobre los "espacios de la muerte" se puede consultar: "Cultura del terror, espacio de la muerte: El Informe Casement sobre el Putumayo y la explicación de la tortura", Primera y Segunda parte, Falsas Riendas. Revista de Arte y Pensamiento, nos 1 y 2 y 3 (octubre-noviembre 1986, diciembre 1987, julio 1988): 15-30, 33-45. 
El siglo XIX comienza a producir ese espesor urbano que vemos hoy en día. De hecho, si tenemos en cuenta esto, que es el espacio roturado por la civilización indígena el que principalmente es ocupado por los españoles, comprenderemos el impacto que pudo generar la introducción de ganado, por cuanto este espacio estaba destinado, como sabemos, a un cierto tipo de cultígenos, entre ellos el maíz.

La queja más frecuente de los indígenas que se encuentra en la documentación a nuestro alcance, era la de que el ganado de los españoles dañaba sus sementeras. Entonces, de cierta manera el ganado estaba robando un espacio para proporcionar proteínas animales, cuando los indígenas ni siquiera estaban consumiendo dichas proteínas. Además, si pensamos que en pastos naturales donde una res más bien flaca necesita cuatro tallas de pastos y que con el tiempo va a necesitar seguramente más, nos daremos cuenta [de] que esto significó un estrechamiento del espacio roturado. Este hecho tuvo que producir un impacto tremendo en las poblaciones nativas asentadas en los intersticios que les dejaban las fundaciones de españoles.

De igual forma podemos imaginar el otro problema que se presentó a los miembros de los grupos étnicos americanos en la sustitución de cultivos. Reemplazar el maíz, que tiene un rendimiento mucho más alto que el trigo, provocó un desgaste mucho mayor en las faenas productivas, frente al incremento de la cobertura espacial que se necesitó para obtener un quantum de trigo similar. De hecho, hoy en día sabemos que los encomenderos inicialmente crearon, a pesar de las tradiciones, los aposentos y que en ellos fueron sustituyendo los cultivos tradicionales de maíz por cultivos de trigo para comercializar en los centros urbanos.

Luego tienen ustedes el problema de las inmunidades. Es un hecho muy conocido que los africanos desde épocas relativamente tempranas crearon inmunidades a enfermedades de cierto tipo; enfermedades eruptivas que por su naturaleza sociocultural poseen una gradación de gravedad específica.

Desde las simples enfermedades o fiebres de los niños, tan corrientes hoy en día, hasta las viruelas que son ya una plaga mayor, tuvieron que ser tratadas no solo por medio de la farmacopea de la época, sino a través de los procesos adaptativos muchos de los cuales aún no han terminado. Cualquier enfermo, si iba, por ejemplo, a Hawái, producía una verdadera catástrofe dentro de los indígenas.

Es así como podemos ver el impacto que necesariamente se produjo con el simple hecho de la llegada de los primeros españoles a este Continente. Observen ustedes que no hay aquí ninguna connotación de tipo moral, ni ningún problema moral en juego. Es sencillamente algo que podemos atribuir a diferencias culturales que producen o pueden producir resultados desastrosos.

Situándonos en otro nivel, el que corresponde a la organización familiar, casi todos los antropólogos están de acuerdo y nos han ayudado a comprender lo que significan las reglas de parentesco; lo que significa un tema como la matrilinealidad, la uxorilocalidad, todo este tipo de fenómenos que los españoles no aceptaron y que 
modificaron profundamente en varios sentidos. Por ejemplo, claramente podemos ver que la línea de sucesión como la impusieron, la patrilinealidad, deja de lado la regla que dentro de los Muiscas era más aceptada: que el que hereda es el hijo del cacique, no el hijo de la hermana del cacique.

De esta manera vemos como se modificaron las reglas para esta tradición, así como también el hecho de que las unidades familiares se vieron afectadas por su división arbitraria, cuando se establecieron las encomiendas. Una comunidad dividida en varias encomiendas y los encomenderos luchando por situar a sus indios encomendados en las cercanías de sus aposentos.

Fue así como estas luchas económicas alteraron las reglas, los patrones y los sistemas de parentesco. Así mismo, el hecho de que las familias se disolvieran por la separación de los indígenas tributarios, en el momento en que eran sometidos a esta obligación, puesto que a su vez se los llevaban para el trabajo en las minas, constituyendo seguramente cuadrillas masculinas que no tenían acceso sexual en la época más propicia para ello, alteraron profundamente las posibilidades de restablecer antiguos equilibrios demográficos y retardaron por más de un siglo la lenta pero sostenida recuperación de la población indígena que hoy día observamos.

Ante esta situación, los cambios que se produjeron dentro de la estructura social son bastante obvios. Debemos estar preparados para abandonar la idea de los manuales que nos hacen suponer que las bases étnicas crearon una pirámide social a cuya cabeza estarían los españoles, luego seguirían los indígenas, para terminar en los esclavos. Los mestizos quedarían situados en algún sitio intermedio, dentro de una gama de posibilidades muy amplias. Esta es una idea muy rudimentaria de lo que ocurrió en una sociedad y en una aristocracia como la colonial. En realidad, esto no es cierto, es obviamente falso; lo que tenemos que pensar son varias cadenas que no tienen necesariamente correspondencia hacia ella en la estructura social. Por ejemplo, entre los españoles es evidente que hubo encomenderos, que hubo beneméritos, que hubo funcionarios, y también una masa de artesanos: artesanos pobres, unidades de estrato medio de artesanos. Inclusive de los oficios artesanales también sabemos que tenían su jerarquía. En la cabeza se colocaban los plateros y en el último lugar, naturalmente, los zapateros. Es un poco patético el asunto; si se colocan en los pies están en los pies.

En el caso del indígena sabemos que la conquista no destruyó completamente la concepción chibcha de la jerarquía de los famosos capitanes y luego la masa del pueblo. Lo mismo en México los macheguales; en fin, personas que tuvieron incluso entre los esclavos, capitanes; esclavos que tenían capataces [...]. Entonces existieron varias jerarquías entre las cuales no hay una absoluta correspondencia por cuanto ocupan ámbitos diferentes pro de todas maneras mucho más complejas de lo que suelen decir los manuales.

Tenemos que pensar en el impacto que produjo el hecho de la alteración de los patrones sociales y económicos a raíz de la forma como se canalizó el excedente eco- 
nómico a través de estas jerarquías. Es decir, sabemos que el excedente económico en las sociedades indígenas se canaliza en esta dirección, pero es una nueva jerarquía la que va a sustituir a la jerarquía indígena, para que el encomendero se apropie del excedente económico-artesanal; entonces, hay una frustración de equilibrios económicos y sociales muy profunda, que se aúna a los otros fenómenos. En este sentido el impacto político es bastante obvio puesto que los españoles, para lograr canalizar el tributo en la cúspide, tenían que preservar unos niveles intermedios, unas ratas o promedios.

Ellos cortaron todo, toda democracia, toda especialización intelectual, llamémosla así, de las sociedades indígenas, de tal manera de procurar que el excedente se canalizara en una forma diferente y naturalmente fortalecer el nuevo poder político.

Quisiera recordar un bello poema, de la inspiración de don Miguel de Portilla [...], en el que un indígena, sacerdote indígena, le dice a un dominicano: "Señor, nuestros antepasados nos enseñaron que hablar mal de los dioses de nosotros es mala educación". Yo creo que el sentido de esta frase, de esta linda inspiración, es el del refinamiento de una cultura, un refinamiento bien sofisticado, enfrentado a otra cultura que es ciega y sorda, y llena de sentido misionero. Creo que esto va a ser más problemático porque la interpretación tradicional nos dice que fue una gran ventaja la conversión de los indígenas [...]. Ya vemos a qué precio; fueron millones de indígenas los que desaparecieron. Me parece que es bastante problemático el pensar en el tipo de desplome interior que se traduce en las sociedades indígenas por cuanto sentimientos y valores culturales se ven atacados de raíz. Pero realmente, y creo que vale la pena analizarlo, que hay que establecer cómo se produjo ese desplome interior, qué mecanismos se jugaron en ese deslome interior. Cómo, por ejemplo, el hecho de que si el sacerdote indígena establecía el ritmo de las cosechas y la comunidad estaba asentada en ese saber, ese sacerdote se vio contradicho y se vio anulado por la ortodoxia que impuso un dogma que condena y castiga a la superstición. Entonces, cómo se priva a una sociedad de sistemas culturales, mentales, etcétera.

Sin embargo, pienso que todavía nos puede dar mucho el saber que el problema radica en que todavía, hasta ahora, hasta qué punto, el que está escribiendo, el que está utilizando una serie de conceptos españoles como el de "miserable", tiene un sentido y un dejo de originalidad. 